\title{
The influence of indenter bluntness on the apparent contact stiffness of thin coatings
}

\author{
A.M. Korsunsky ${ }^{\mathrm{a}}, \mathrm{A}$. Constantinescu ${ }^{\mathrm{b}, *}$ \\ a Department of Engineering Science, University of Oxford, Parks Road, Oxford OX1 3PJ, England, United Kingdom \\ b Laboratoire de Mécanique des Solides, (CNRS UMR 7649, Polytechnique, Mines, Ponts and Chaussées), 91128 Palaiseau cedex, France
}

In the present paper, the influence of punch tip sharpness on the interpretation of indentation measurements is considered.

Firstly, in order to obtain analytical insight into the nature of the problem, closed form solutions are presented for the indentation of a homogeneous elastic half-space by an axisymmetric indenter of arbitrary shape, including Hertzian, conical, and conical indenter with a rounded tip. Next, a fast and efficient numerical implementation of a semi-analytical approach to the solution of problems about frictionless axisymmetric indentation of an elastic layer on a dissimilar substrate is described. The approach allows rapid determination of the load-displacement curves for an arbitrary punch shape. The aim of the study was to explore the implications of a finite indenter tip radius for the problem of property identification of thin films. The variation of the apparent substrate stiffness with indentation depth was established for several generic indenter shapes, namely for flat, conical and parabolic punches, and for a conical punch with a rounded tip. It is demonstrated that in each of these cases the depth variation can be described by a simple curve belonging to a family of two-parametric functions. On the basis of these findings we assess the efficiency of using different punch shapes for property determination. A procedure is proposed for this purpose which relies on the use of the depth variation profiles established here. Finally, the influence of imperfect punch shape on the accuracy of analysis is considered.

\section{Introduction}

Modern surface engineering relies on the use of thin coatings to achieve the required improvements in the stiffness, hardness, friction, scratch and wear resistance over the uncoated material. Although a variety of approaches are being developed, direct measurement of the coating's mechanical characteristics is often difficult. Frequently the key properties are assessed indirectly from indentation experiments.

In essence, the task objective of the indentation experiment is then to 'back out' the desired parameters (hardness, modulus) for the system (coating in particular) by interpreting the data obtained from continuous recording indentation testing (CRIT). However, even when sample response can be assumed purely elastic, this inverse problem (indentation interpretation) remains challenging. As in most cases of inverse problems, the direct problem has to be addressed first.

The main difficulty of modelling the indentation experiment stems from the nature of the contact boundary condition between the indenter and the sample. The contact condition is expressed in the form of an inequality that expresses mutual non-interpenetrability of the indenter and sample. Otherwise the problem may be thought as a mixed boundary value problem (part displacement, part traction

\footnotetext{
* Corresponding author.

E-mail addresses: alexander.korsunsky@eng.ox.ac.uk (A.M. Korsunsky), constant@lms.polytechnique.fr (A. Constantinescu).
}

boundary conditions) with unknown boundary that may move and is determined by both the applied load and the substrate deformation response. These effects result in problem non-linearity even in the case of a linearly elastic sample. Furthermore, the problem becomes more and more difficult with progressively thinner coatings.

Nevertheless, for some contact problems, complete solutions of the indentation problem have been obtained analytically, as described in general reviews of the classical results on the subject in [1,2]. Literature reviews indicate that closed form elastic contact solutions for coated systems (see for example [3-5]), or finite element calculations for elastoplastic systems using the finite element method (see for example the comparison of 2D and 3D computations by Lichinchi et al. [6]) show that they are difficult to obtain even when all material properties are known. This ensures that in practice parametric studies for the identification of material properties are outside a practical reach.

In order to overcome these difficulties, in the last decades a series of simple response functions have been proposed that relate directly the measured indentation depth and force to the material parameters. A series of papers in the case of homogenous half-spaces are based on the pioneering results by Tabor [7] and more recently by Doerner and Nix [8] or Oliver and Pharr [9].

In the paper we propose a simplified response function for the indentation of an elastic coated system. The proposed response function uses relative indentation depth (i.e. indentation depth 


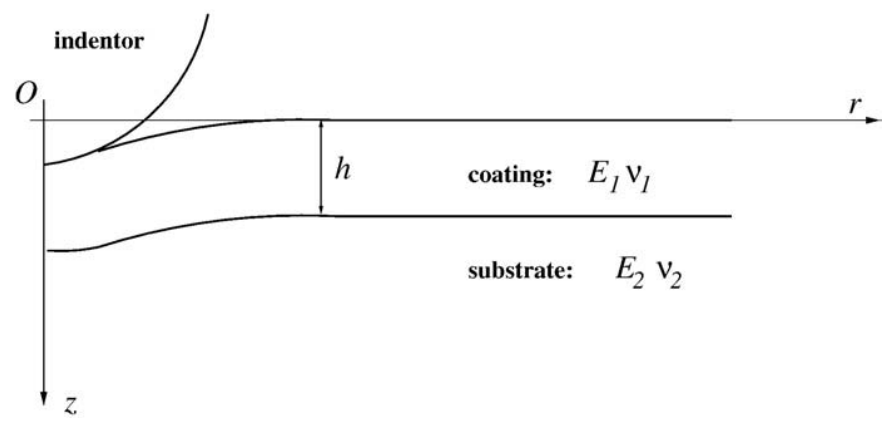

Fig. 1. A schematic diagram of the coated system during indentation.

divided by the coating thickness) as the argument, and aims to compute the indentation force using the elastic properties of the coating and substrate as parameters. In fact, the model response function proposed here represents the apparent elastic modulus. This is achieved by normalising the indentation force by the indentation solution corresponding to a homogenous half-space.

In order to assess the efficiency of representation achieved by the model response function, a comparison is made with detailed numerical simulation results. Numerical solutions were obtained for frictionless indentation of an layered half-space by punches of practically relevant geometries (flat punch, Hertzian indenter, cone, and cone with a tip blunted to a sphere or parabola), representing the more realistic indenter tip shape. The mathematical implementation of the desired solutions is achieved by solving an integral equation using the method proposed by Lebedev and Uflyand [10], and previously utilised and reported by Yu et al. [5]. The selection of this method from a series possible solutions $[4,3]$ is motivated by the ease of programming for a large class of indentor shapes, and acceptable stability of the solution even for extreme values of layer thickness, i.e. the cases of very thin or thick coating.

The experimental results for hardness have been obtained from the data on nanoindentation of thin coatings on tool steels [11].

The present paper introduces and validates the use of the proposed response function. The parametric study carried out here

- shows that for all considered indentor shapes the apparent elastic modulus can be represented in a compact and efficient manner by a two-parameter function;

- provides estimates of the elastic moduli of the coating from a small number of measurements, and indicates the range of relative indentation depths needed for obtaining reliable assessments of coating properties;

- provides estimates of measurement errors arising from the use of blunted (rounded) conical indenters when the analysis is carried out as if the indenter were a perfect cone. The blunting radius may also be estimated from the measurement results.
It follows that the model response function can be used for the identification of elastic moduli, and provides significant simplification of laborious computations required for the underlying direct and inverse problems [12].

It is worth noting that the present analysis is focused on the consideration of elastic deformation of the coating and substrate in response to indentation by axisymmetric rigid indenters. The limitations of such an approach are apparent, and can be identified up front. Firstly, in real indentation experiments involving almost all classes of materials, the response of the material contains the combination of elastic and plastic behaviour. Although for ideally sharp indenters the onset of plasticity is immediate, in practice some degree of indenter bluntness is always present, and, in fact, requires careful calibration [13]. At low indentation loads (and indentation depths) it is conventional to identify the regime where the response is dominated by elasticity. At higher loads and deeper penetrations plasticity becomes the dominant deformation mechanism.

The linear elastic models considered in the present paper have their applicability clearly delimited by the onset of plasticity within the coating or the substrate. It is apparent that, whatever the material considered, plastic effects will come for the fore at certain stage in the indentation experiment; in the notation used in this paper, as the relative indentation depth (RID) becomes greater. It should therefore be understood that model predictions for large RID values (RID $\gg 1$ ) can only be viewed as extrapolations of the linear elastic model, for the case of ideally elastic material. In practice, deviations from these predictions due to plastic flow are expected to manifest themselves.

The value of the proposed approach, nevertheless, lies in the clear identification of the trends and interrelationships between test parameters in the indentation experiment. For example, it is apparent that even in the case of purely elastic response evaluation of the coating-only properties must take into account the substrateindenter interaction, via the intermediate of the coating layer. Furthermore, limits of resolution and sensitivity of the system can be clearly identified.

\section{A simple response function for the apparent contact modulus}

The material parameter that governs the indentation response of elastic homogeneous substrates can be referred to as the contact modulus, also known as the plain strain modulus. It is defined as:

$E^{*}=\frac{E}{1-v^{2}}$.

In the present study we assume that the indenter is rigid and undeformable. In cases when the indenter tip deformation needs to be taken into consideration, a combined contact modulus should be defined in an appropriate way.

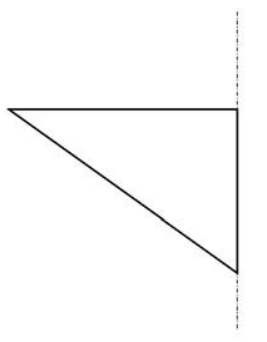

conical

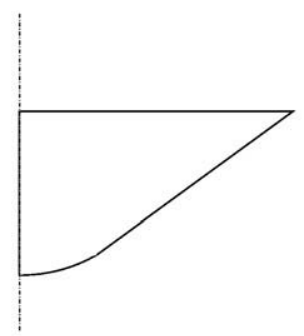

blunted cone

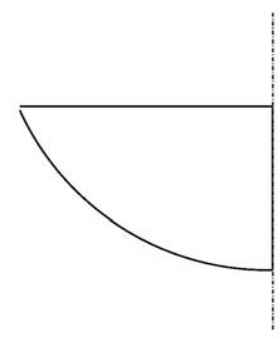

spherical

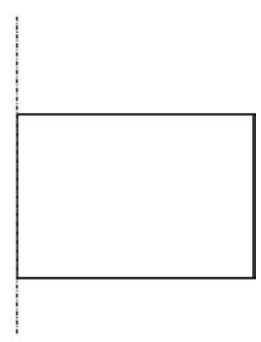

flat

Fig. 2. Different shapes of indenters considered in this paper. 
For the coated system, the contact modulus appearing in Eq. (1) can be expressed directly as a function of the applied load $P$, the displacement of the indenter $d$ and the contact radius $a_{H}(d)$ for the equivalent homogeneous substrate problem:

$E^{*}=\frac{E}{1-v^{2}}=\frac{P}{2 d a_{H}(d)}$.

General solution techniques using integral transform methods for frictionless elastic contact mechanics problems for homogeneous substrates were described by Sneddon. The functional dependence of the contact radius $a_{H}$ on the indenter displacement $d$ for important indenter geometries, such as the cone and sphere, is found to have the form of a power law. This dependence, $a=a_{H}(d)$, can be used to eliminate the contact radius from the expression for the contact modulus. Consequently, $E^{*}$ can be defined in terms of the applied load and indenter displacement.

For a coated system the apparent contact modulus is therefore defined as:

$E^{*}(d)=\frac{P}{2 f d a_{H}(d)}$.

For coated systems, the apparent contact modulus varies with the indentation displacement $d$. It is important to stress again that the above definition contains the function $a_{H}(d)$ which is derived from the solution for the given indenter shape upon a homogeneous substrate, and not the real contact radius $a$. The dimensionless parameter $f$ appearing in the above expression is a number calculated from the punch shape alone, in a manner defined in the following section.

The solution for the contact radius as a function of the applied force or indenter displacement is not available in closed form for the case of coated systems. Therefore, a closed form expression for the apparent contact modulus cannot be obtained. This parameter is, however, of great importance for the purposes of experimental indentation analysis using depth sensing indentation, since it provides a measure of the composite response of the substrate-coating system in terms of the parameters directly recorded by the testing system.

The purpose of this paper is to present the numerical results for the apparent contact modulus for elastic coated systems, and to give a simple representation of the apparent contact modulus as a function of the indentation depth (Fig. 1).

We show that for coated systems a good approximation for the entire family of numerical results is given by the following proposed model response function [11]:

$E^{*}(d)=e_{2}^{*}+\frac{e_{1}^{*}-e_{2}^{*}}{1+\left(\frac{1}{\beta_{0}} \frac{d}{h}\right)^{\eta}}$.

Here $h$ is the film thickness, and $e_{1}^{*}, e_{2}^{*}, \beta_{0}$ and $\eta$ are positive constants to be determined from fitting. It is clear from the above formula that $e_{2}^{*}$ represents the apparent contact modulus obtained in the extreme of very deep indentation. Similarly, $e_{1}^{*}$ is the apparent contact modulus found from very shallow indentation. The significance of all parameters is discussed further in the text.

\section{The elastic indentation problem}

In order to analyse a series of indentation problems and check the validity of the proposed model function, we have chosen to compute the solution of the elastic indentation problem using a boundary

Table 1

Different combinations of Young's moduli and Poisson's ratios used in the computations, and the corresponding plane strain moduli of the substrates and layers.

\begin{tabular}{lllll}
\hline$v$ & $E$ & $E^{*}$ & $E$ & $E^{*}$ \\
\hline 0.2 & 96 & 100 & 480 & 500 \\
0.3 & 91 & 100 & 451 & 500 \\
0.4 & 84 & 100 & 420 & 500
\end{tabular}

integral approach proposed by Yu et al. [5]. To illustrate the basis of the solution procedure and provide some confidence in the validity of the proposed numerical solution in the sequel we shall sketch the main steps of the solution proposed by Yu et al. [5] and describe the main features of our numerical implementation. We may further note that the symbolic form of the equations can be readily obtained using the Tensor2Analysis and Mathematica package defined in Constantinescu and Korsunsky, 2007 [18].

Let us consider an elastic layer of thickness $h$ (designated by the subscript $j=1$ ) lying on a dissimilar semi-infinite elastic substrate (designated by the subscript $j=2$ ). The elastic solution can be expressed in the cylindrical coordinate system as:

$2 \mu_{j} u_{r j}=-\varphi_{j, r}-z \psi_{j, r}$

$2 \mu_{j} u_{z j}=-k_{j} \psi_{j}-\varphi_{j, z}-z \psi_{j, z}$

$\sigma_{z z j}=2\left(1-v_{j}\right) \psi_{j, z}-\varphi_{j, z z}-z \psi_{j, z z}$

$\tau_{r z j}=\left(1-2 v_{j}\right) \psi_{j, r}-\varphi_{j, r z}-z \psi_{j, r z}$

where $u_{r j}, u_{z j}$ are the components of the displacement vector and $\sigma_{z j}$, $\sigma_{r z j}$ are the components of the stress tensor. These elastic fields can be expressed in terms of a pair of harmonic Papkovich-Neuber potentials $\psi_{j}, \varphi_{j}, j=1,2:^{1}$

$$
\begin{aligned}
& \psi_{1}(r, z)=\int_{0}^{\infty}\left(A_{1} \cosh \lambda z+A_{2} \sinh \lambda z\right) \frac{J_{0}(\lambda r)}{\sinh \lambda h} d \lambda \\
& \varphi_{1}(r, z)=\int_{0}^{\infty}\left(A_{4} \cosh \lambda z+A_{3} \sinh \lambda z\right) \frac{J_{0}(\lambda r)}{\sinh \lambda h} d \lambda \\
& \psi_{2}(r, z)=\int_{0}^{\infty} A_{5} \exp (-\lambda(z-h)) J_{0}(\lambda r) d \lambda \\
& \varphi_{2}(r, z)=\int_{0}^{\infty} A_{6} \exp (-\lambda(z-h)) \frac{J_{0}(\lambda r)}{\lambda} d \lambda .
\end{aligned}
$$

The unknown functions appearing in the potential formulation above can be determined from the boundary conditions at the surface $z=0$ :

$u_{z 1}(r, 0)=d-\delta(r)(0 \leq r \leq a)$

$\sigma_{z z 1}(r, 0)=0(a \leq r \leq \infty)$

$\sigma_{r z 1}(r, 0)=0(0 \leq r \leq \infty)$.

The conditions at the film-substrate interface, $z=h, 0 \leq r \leq \infty$, depend on the nature of bonding. For a perfectly bonded film these are

$$
\begin{aligned}
& u_{z 1}(r, h)=u_{z 2}(r, h) \\
& u_{r 1}(r, h)=u_{r 2}(r, h) \\
& \sigma_{z z 1}(r, h)=\sigma_{z z 2}(r, h) \\
& \sigma_{r z 1}(r, h)=\sigma_{r z 2}(r, h)
\end{aligned}
$$

and for a freely sliding film they are:

$$
\begin{aligned}
& u_{z 1}(r, h)=u_{z 2}(r, h) \\
& \sigma_{z z 1}(r, h)=\sigma_{z z 2}(r, h) \\
& \sigma_{r z 1}(r, h)=\sigma_{r z 2}(r, h)=0 .
\end{aligned}
$$

\footnotetext{
${ }^{1}$ For a general presentation of displacement potentials see [14].
} 

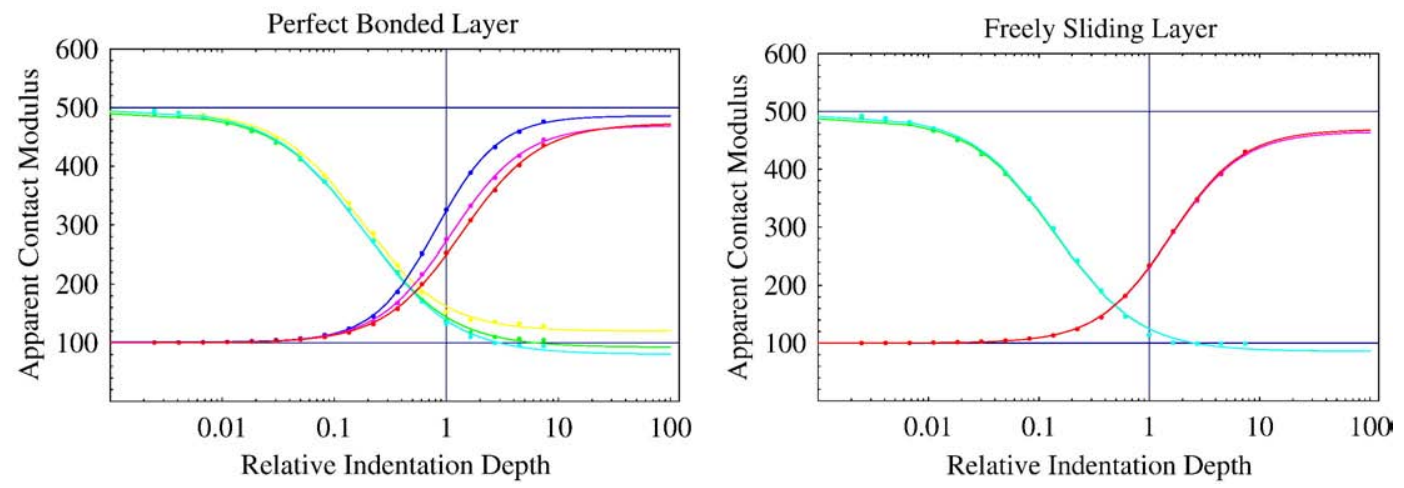

Fig. 3. The apparent contact modulus versus relative indentation depth for perfectly bonded and freely sliding film respectively, indented with a cone.

The determination of the elastic fields now requires the determination of the unknown functions $A_{i}(\lambda), i=1,6$. This is accomplished in two steps.

Firstly, the boundary conditions at the interface are used together with the condition (15) to express all $A_{i},(i=1,6)$ in terms of $A_{1}$.

Secondly, the sought function $A_{1}$ is expressed in terms of another unknown function, $H$, as follows

$A_{1}(\lambda)=[1-g(\lambda)] \sinh \lambda h \int_{0}^{a} \frac{2 \mu_{1} d}{\pi\left(1-v_{1}\right)} H(t / a) \cos \lambda t d t$.

As a consequence, the surface contact conditions $(13,14)$ are now expressed as the following single integral equation

$H(\tau)-\frac{1}{\pi} \int_{0}^{1} M(y, \tau) H(y) d y=F(\tau), \quad(0 \leq \tau \leq 1)$.

The parameter $\tau$ represents the normalised radial coordinate within the patch of contact, $\tau=t / a$. The terms in the above equation relate to some specific aspects of the contact problem. Function $F(\tau)$ depends solely on the indenter shape and the indentation depth. The kernel $M(y, \tau)$ depends on the elastic properties of the layer and substrate, the thickness of the former, and the contact radius $a$. It is defined by $M(y, \tau)=K(y+\tau)+K(y-\tau)$, where

$K(u)=\frac{a}{h} \int_{0}^{\infty} g(w) \cos \left(\frac{a u w}{h}\right) \mathrm{d} w$,

where $h$ is the layer thickness, and $a$ is the contact radius.

We note for the future that the key computational step employed in the solution concerns the evaluation of integral in Eq. (25). It should be reported that, particularly for thin coatings, the kernel of this integral becomes oscillatory, and requires careful treatment. This is particularly important since the integral needs to be evaluated repeatedly, particularly so in the case of incomplete contacts, when the extent of the contact patch needs to be found by iteration.

The approach adopted to the evaluation of this integral involved (i) identifying the "tail" where the behaviour of the kernel is monotonic and in fact allows analytical evaluation; (ii) use of special adaptive quadrature in Mathematica [16] to evaluate the oscillatory part of the integral, and (iii) implementation of the resulting module in a compiled form, allowing faster computation. All these measures together resulted in adequately good performance and efficient evaluation of the integral in question (see below).

The function $g$ appearing in Eq. (23) and the expression for the kernel (25) is non-singular, and depends on the combination of elastic properties of the layer and substrate, and on the conditions at the interface. The expressions for $g(w)$ in [5] for the perfectly bonded layer contain a typographical error. The authors of that paper have kindly provided with a correction upon our request. We present the correct formulae in Appendix A.

In particular, in the case of a layer perfectly bonded to a substrate of identical material it is found that $g(w)=0$. Eq. (25) for the kernel must then be understood in the generalised function sense, and yields a constant value, which results in the integral equation kernel $M(y, \tau)$ being identically equal to zero. The homogeneous substrate solution is therefore given by $H(\tau)=F(\tau)$.

Cases when incomplete contacts are considered (i.e. the extent of contact is not known a priori from the punch shape) introduce a nonlinearity in the formulation, and force the corresponding solution procedure to be iterative. However, even in this case important computational savings can be achieved if the lack of coupling between the elastic properties and the punch shape is explored.

The punch shape can be defined with respect to its tip, i.e. the point of first contact, which for the axisymmetric case will be assumed to lie on the axis of rotational symmetry. A function of the form $\delta(r / a)$, $\delta(0)=0$ was used by Yu et al. [5] to define the punch shape. We use a different convention here, writing $\delta(r), \delta(0)=0$. This formulation is preferred since the punch shape is manifestly independent of the contact radius $a$, which is generally not known a priori. The only modification that ensues from this choice is that the function $F(\tau)$ appearing in the right hand side of Eq. (24) is now given by

$F(\tau)=1-\frac{a \tau}{d} \int_{0}^{\pi / 2} \delta^{\prime}(a \tau \sin \theta) \mathrm{d} \theta$

Finally, the total indentation load is obtained by integrating the pressure distribution under the indenter, and is given by

$P=2 a d E_{1}^{*} \int_{0}^{1} H(\tau) \mathrm{d} \tau$

where $E_{1}^{*}=E_{1} /\left(1-v_{1}^{2}\right)$ is the plane strain modulus of the coating.

The solutions for frictionless axisymmetric indentation of a homogeneous substrate can be obtained analytically in closed form for a

Table 2

Computed apparent contact moduli for the indentation of a perfectly bonded layer with a cone.

\begin{tabular}{lllllcll}
\hline$v_{1}$ & $E_{1}^{*}$ & $v_{2}$ & $E_{2}^{*}$ & $e_{1}^{*}$ & $e_{2}^{*}$ & $\beta_{0}$ & $\eta$ \\
\hline 0.2 & 500 & 0.4 & 100 & 493. & 119. & 0.174 & 1.181 \\
0.3 & 500 & 0.3 & 100 & 490. & 92. & 0.180 & 1.117 \\
0.4 & 500 & 0.2 & 100 & 495. & 80. & 0.186 & 1.085 \\
0.2 & 100 & 0.4 & 500 & 101. & 485. & 0.813 & 1.549 \\
0.3 & 100 & 0.3 & 500 & 100. & 469. & 1.107 & 1.329 \\
0.4 & 100 & 0.2 & 500 & 99. & 473. & 1.375 & 1.255 \\
\hline
\end{tabular}


Table 3

Computed apparent contact moduli for the indentation of a freely sliding layer with a cone.

\begin{tabular}{llllllll}
\hline$v_{1}$ & $E_{1}^{*}$ & $v_{2}$ & $E_{2}^{*}$ & $e_{1}^{*}$ & $e_{2}^{*}$ & $\beta_{0}$ & $\eta$ \\
\hline 0.2 & 500 & 0.4 & 100 & 493 & 85. & 0.140 & 1.143 \\
0.3 & 500 & 0.3 & 100 & 488. & 86. & 0.141 & 1.145 \\
0.4 & 500 & 0.2 & 100 & 493. & 85. & 0.140 & 1.143 \\
0.2 & 100 & 0.4 & 500 & 100. & 468. & 1.557 & 1.331 \\
0.3 & 100 & 0.3 & 500 & 100. & 464. & 1.551 & 1.332 \\
0.4 & 100 & 0.2 & 500 & 100. & 468. & 1.557 & 1.331 \\
\hline
\end{tabular}

punch of arbitrary shape [2,17]. As noted above, in this case $H(\tau)=F(\tau)$. The condition of smooth contact at the contact perimeter assumes the form $F(\tau)=0$ (note that this condition does not generally hold for a layered system). The relationship between the indenter displacement $d$ and the contact radius $a_{H}$ can therefore be found explicitly by solving Eq. (26) with respect to $a$. The homogeneous substrate solution is therefore characterised by this functional dependence of contact radius on indenter displacement, $a=a_{H}(d)$, and the following value of the indentation load:

$P_{H}(d)=2 d a_{H}(d) E_{1}^{*} \int_{0}^{1} F(\tau) \mathrm{d} \tau$

This formula is the basis of the apparent contact modulus definition given in the introduction. Indeed, for indenter of any chosen shape on a layered material it is now possible to normalise the solution for the coated system with respect to the values for homogeneous material.

The application of this analysis to the interpretation of modelling and experimental results is as follows. Let the indenter shape be known, and a pair of indenter displacement and load values be obtained in some way, by measurement or calculation. Based on Eq. (28), the apparent contact modulus can be computed by

$E^{*}=\frac{P}{2 f d a_{H}(d)}=\frac{P}{2 d a_{H}(d)}\left[\int_{0}^{1} F(\tau) d \tau\right]^{-1}$.

Note that for the evaluation of this parameter, only the knowledge of the displacement-load pair of values is required, and that of the contact radius-indenter displacement function $a_{H}(d)$ for the homogeneous solution. The parameter $f$ defined implicitly in the above expression is

$f=\int_{0}^{1} F(\tau) d \tau=1-\frac{a_{H}}{d} \int_{0}^{1} \tau \mathrm{d} \tau \int_{0}^{\pi / 2} \delta^{\prime}(a \tau \sin \theta) \mathrm{d} \theta$.

For example, for the flat punch $\delta^{\prime}=0$, and hence $f=1$. Using the results of the following section for $a_{H}(d)$, it is easy to establish that for the cone $f=1 / 2$, and that for the Herztian indenter $f=2 / 3$.
The numerical implementation of the system of equations described above has been programmed in Mathematica [16]. The solution was found through an iterative procedure.

A Gauss-Legendre quadrature rule and the roots of Legendre polynomials as collocation points were used for the representation of the function $H$, as well as for computing the kernel $M$. This approach allows Eq. (24) to be transformed into a linear algebraic system which can be easily solved for a given value of the contact radius $a$.

The order of approximation for Eqs. (25) and (24) was varied to establish convergence, the value of 30 being usually sufficient for both. Augmenting the number of collocation points above 30 does not necessary enhance the accuracy of the solution, for reasons associated with the deteriorating accuracy of Legendre interpolation. As a consequence, the kernel computed with a large number of points may be close to singular.

The correct value of the contact radius is found by imposing the condition of vanishing contact pressure at the edge of the contact region: $\sigma_{z z 1}(a / h)=0$, i.e. $H(1)=0$ using our representation of the solution. This is done using an iterative procedure using e.g. the dichotomy method, starting with an initial value corresponding to the contact radius in the case of a homogenous substrate.

In order to speed up the numerical computations we have used one of the memory control options of the Mathematica programme (f[x_] := $\mathrm{f}[\mathrm{x}]=\ldots$ ) to avoid repeated computations of the same expression, for example of the integral equation kernel (25). We also used the automatic compilation procedure (Compile) in order to speed up the evaluation of functional calls. The combined use of these options improved the computing time by a factor of 20 .

The accuracy of the solution procedure was tested by comparison with the well known analytical solution for the homogenous substrate.

\section{Results and discussion}

In the sequel we shall present a series of numerical results for the indentation of perfectly bonded or freely sliding films on a substrate with frictionless indenters of different shapes (Fig. 2). The chosen values of the elastic properties for the substrate and the moduli are shown in Table 1, which shows that both stiff on compliant and compliant on stiff layer-substrate configurations were considered.

In the following figures we present the evolution of the apparent contact modulus with the relative indentation depth. We compare the computed values of the apparent contact modulus, denoted by solid dots, with the model response function (4), found by fitting these data, and represented by a continuous line. The least-squares fitting algorithm used was the standard LevenbergMarquardt method implemented within Mathematica (function NonlinearFit).
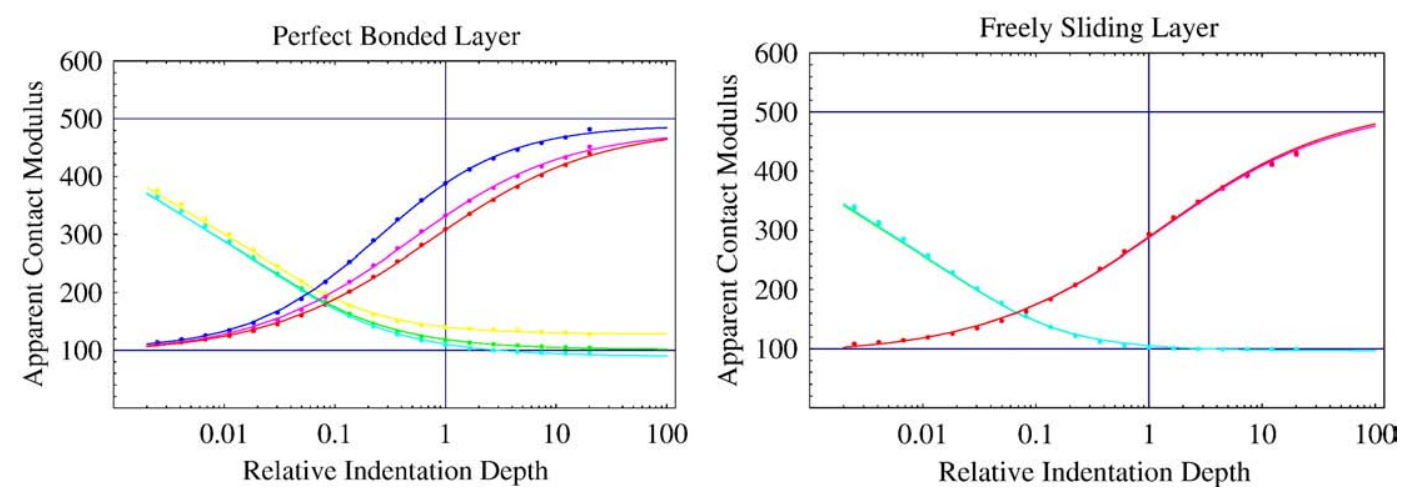

Fig. 4. The apparent contact modulus versus relative indentation depth for perfectly bonded and freely sliding film respectively, indented with a sphere of radius $R=0.1$. 

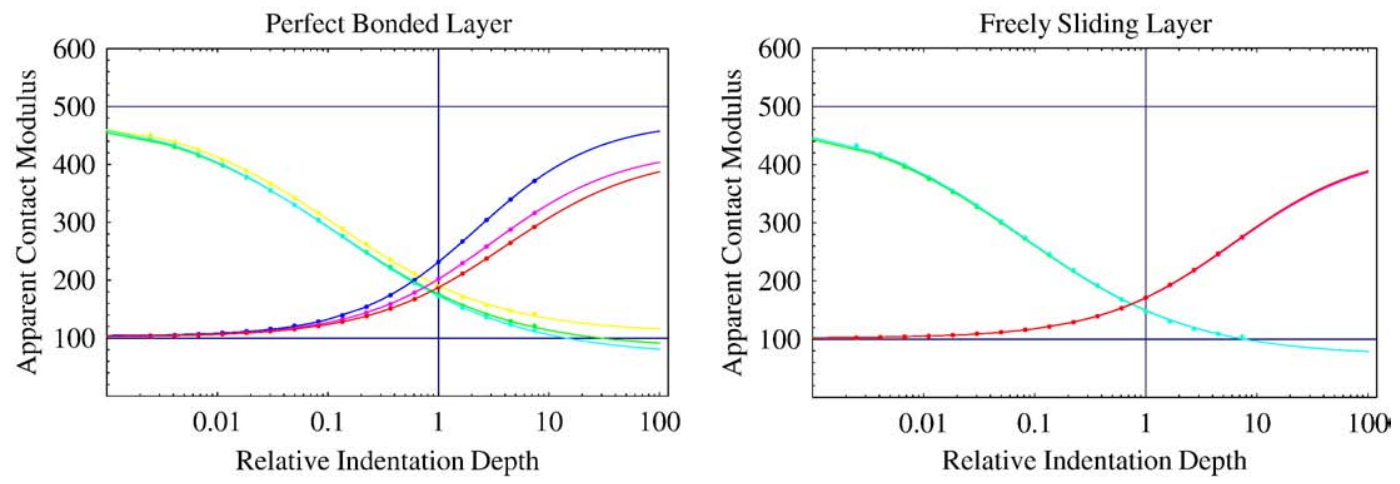

Fig. 5. The apparent contact modulus versus relative indentation depth for perfect bonded and respectively a freely sliding film indented with a sphere of radius $R=1$.

Please note that in all figures the same set of coating-substrate parameter combinations is used, as listed in Table 1. Individual legends are therefore omitted to avoid crowding the graphs.

\subsection{Conical indenter}

The conical indenter shape presents special significance for indentation problems, since it is the only axially symmetric punch shape which does not possess an inherent length scale. As a consequence the indentation problem for a homogeneous substrate becomes self-similar. From this result it follows immediately that the load for indentation into homogeneous substrate must be proportional to the contact area. We have already mentioned in the preceding discussion the function $a_{H}(d)$ that describes the relationship between the contact radius and the indenter displacement into homogeneous material. This function is fixed for any given indenter shape. It is easy to demonstrate that for the cone this function is linear, and depends solely on the cone half-angle.

Indeed, for the cone of half-angle $\alpha$, the indenter shape is given by

$\delta(r)=r \cot \alpha$,

and hence the right side of Eq. (26) is

$F(\tau)=1-\frac{\pi}{2} \frac{a \tau}{d} \cot \alpha$.

The contact radius is found by requiring $F(1)=0$, and hence for the conical indenter the contact radius is given by the linear function of indenter displacement,

$a_{H}(d)=\frac{2 d}{\pi} \tan \alpha$.

It also follows from the application of Eq. (30) that $f=1 / 2$ for the cone.

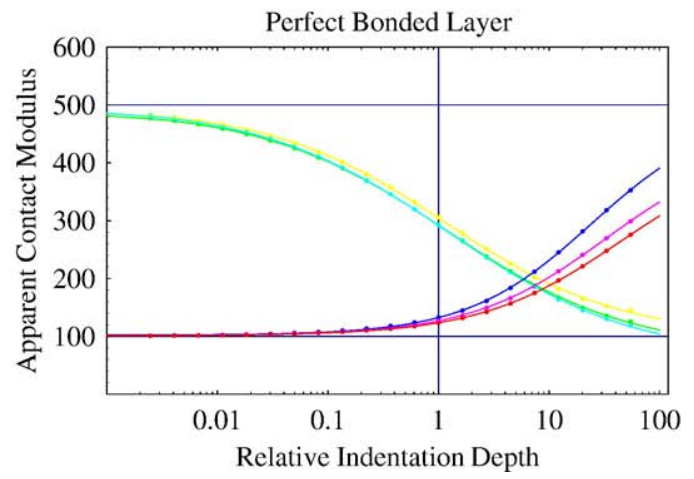

Therefore the apparent contact modulus is defined for the cone as:

$E_{\text {cone }}^{*}(d)=\frac{P}{2 f d a_{H}(d)}=\frac{\pi}{2} \frac{P}{d^{2}} \cot \alpha$.

For the homogeneous substrate, the indentation load is proportional to the square of the indenter displacement. The contact modulus, which is still defined by Eq. (29), is independent of the indentation depth and load.

For the coated system, the contact radius increases with applied load, and the region of significant deformation gradually extends across the layer and into the substrate. The dependence of the contact modulus and contact radius on the displacement deviates from the simple forms found for the homogeneous case. The apparent contact modulus undergoes a transition from the value characteristic for the coating to that for the substrate (Fig. 3). The transition zone depends on the Poisson ratios of the two materials and the boundary condition between the layer and the substrate. In all cases the transition is well represented by the model response function (4).

An inspection of the transition zone indicates that the apparent contact modulus gives a good estimate of the layer plain strain modulus up to relative indentation depth of 0.05 for a compliant layer. For a stiff layer the relative indentation depth of 0.1 already leads to an error exceeding $10 \%$. However, the values estimated using the model response function (see Tables 2 and 3 ) are always within 2\%-10\% of the real values. The large error in the case of the compliant layer comes from the nature of the problem, since the apparent contact modulus even at the relative indentation depth of 10 still shows a $10 \%-20 \%$ difference from the target value.

\subsection{Spherical indenter}

Spherical indenters are particularly important for the determination of elastic properties of materials (compared e.g. with conical

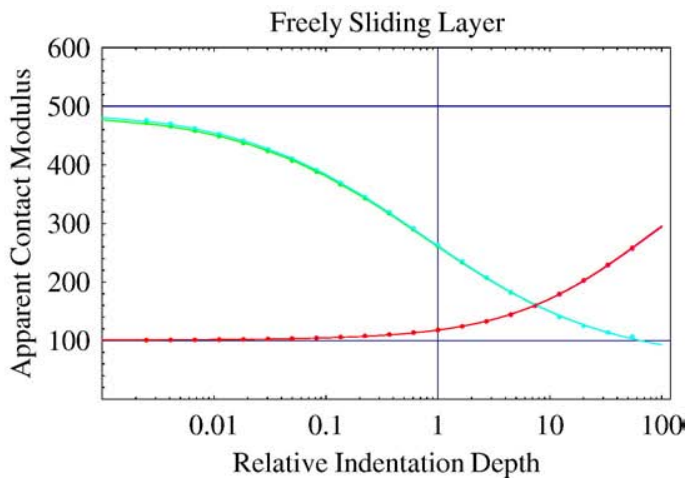

Fig. 6. The apparent contact modulus versus relative indentation depth for perfectly bonded and freely sliding film respectively, indented with a sphere of radius $r=10$. 


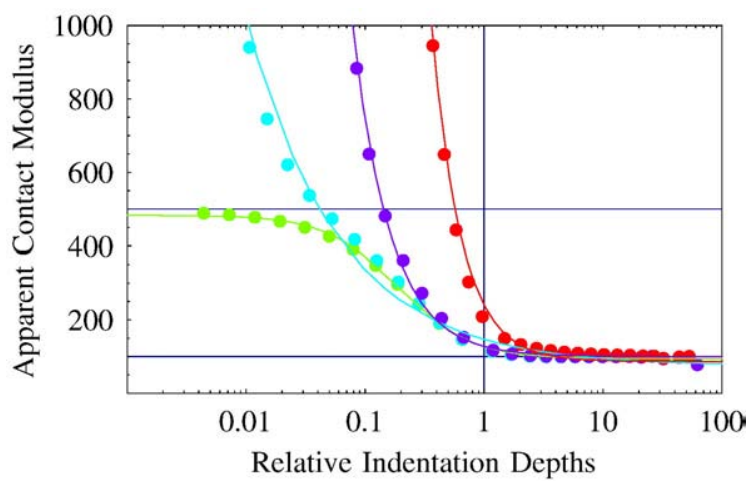

Fig. 7. The apparent contact modulus versus relative indentation depths for a freely sliding film indented with a perfect cone, and with blunted cones with blunt spot radii $b=0.1,1,10$.

indenters), since the former do not immediately induce plasticity at the very early stages of the indentation process.

The shape of the spherical indenter given to the first significant order of approximation by:

$\delta(r)=\frac{r^{2}}{2 R}$

and consequently the right hand side in (24) is

$F(\tau)=1-a^{2} \tau^{2} / R d$

where $R$ is the indenter radius. It also follows from the application of Eq. (30) that $f=2 / 3$ for the Hertzian indenter.

The contact radius is given for a homogenous system by:

$a_{H}(d)=(R d)^{\frac{1}{2}}$,

and therefore the apparent contact modulus is defined as follows:

$E_{\text {sphere }}^{*}(d)=\frac{P}{2 f d a_{H}(d)}=\frac{3 P}{4}\left(\frac{1}{R d^{3}}\right)^{\frac{1}{2}}$

In the case of spherical indenters with different radii, the numerical examples presented in Figs. 4-6 show that the apparent contact modulus follows a similar evolution as in the case of the conical indenter, from the value of the layer to the value of the substrate with increasing relative indentation depth. However, the transition zone is extended over a larger range of indentation depths and depends on the sphere radius.

The model response function again describes well the evolution of the apparent contact modulus. The parameter values are given in Tables (5-7).

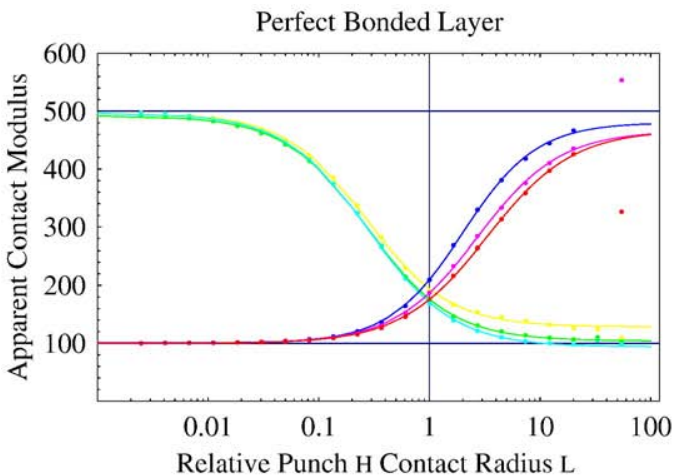

\subsection{Blunted conical indenter}

The case of blunted conical indenters presents a practical interest as experimental indenters are never perfectly conical. The analysis of these indenters is especially important in cases where the film thickness is of the same order of magnitude as the blunting radius of the indenter.

For a conical indenter blunted to the tip radius of $R$ the shape is given by

$\delta(r)=\left\{\begin{array}{lll}\frac{r^{2}}{2 R} & \text { if } & r \leq b \\ \frac{b}{R}(r-b / 2) & \text { if } & r>b .\end{array}\right.$

According to the above definition, the rounded region extends as far as the blunt spot radius of $b$. The cone half-angle $\alpha$ is defined by cot $\alpha=b / R$

The right hand side of Eq. (24) is given by [15]

$F(\tau)= \begin{cases}1-\frac{a^{2} \tau^{2}}{b d} \cot \alpha & \text { if } a \tau \leq b 1 \\ -\frac{a^{2} \tau^{2}}{b d} \cot \alpha\left(1-\sqrt{1-\frac{b^{2}}{a^{2} \tau^{2}}}+\frac{b}{a \tau} \arccos \frac{b}{a \tau}\right) & \text { if } a \tau>b .\end{cases}$

Asymptotic analysis of the above equation shows that for small contact radii function $F$ is identical with that for the Hertzian indenter with tip radius $R=\cot \alpha / b$, while for large contact radii the function $F$ of Eq. (31) emerges, characteristic of the conical indenter.

In practice it is often very difficult to determine precisely the degree of blunting of a nominally sharp conical or pyramidal indenter. Consequently it is common to ensure that the blunting radius does not exceed a certain allowed value, and interpretation is carried out by assuming that the indenter is infinitely sharp. Unfortunately, when this approach is applied to very thin coatings, it may be the source of significant error. Using our solution we can assess the error in estimating the apparent contact modulus that results from assuming that the indenter is infinitely sharp.

Following the logic of measurement interpretation described above, it is natural to define the apparent contact modulus in the same way as for the cone, i.e.

$E_{\text {blunt }}^{*}(d)=E_{\text {cone }}^{*}(d)=\frac{P}{2 f d a_{\text {Hcone }}}=\frac{\pi}{2} \frac{P}{d^{2}} \cot \alpha$.

The consequences of adopting this definition are illustrated in Fig. 7. The values of apparent contact modulus interpreted as described are compared with those obtained using the results of indentation using a perfectly sharp cone. It is clear that even a degree of blunting as small as $b=0.1$ results in severe deviation from the perfect cone results at shallow loads, while the use of even blunter indenters results in massive overprediction of the apparent contact modulus.

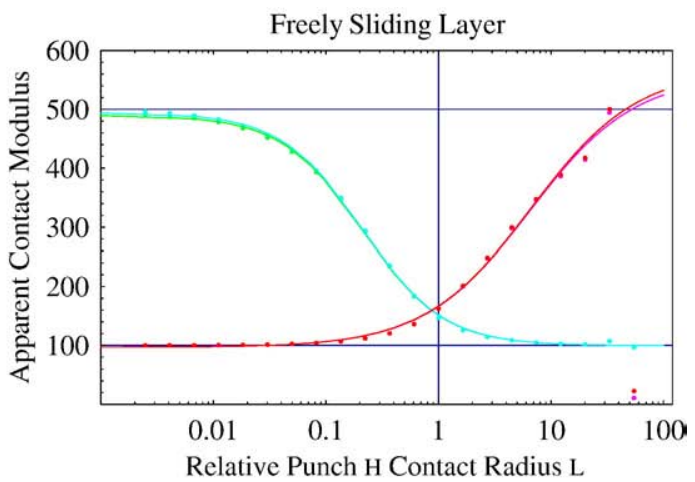

Fig. 8. The apparent contact modulus versus relative contact radius for a perfectly bonded and a freely sliding film respectively, indented with a series of flat punches. 
Table 4

The apparent contact moduli for a freely sliding layer computed from the dependence of load on the relative indentation radius for a flat punch.

\begin{tabular}{llllrrrl}
\hline$v_{1}$ & $E_{1}^{*}$ & $v_{2}$ & $E_{2}^{*}$ & $e_{1}^{*}$ & $e_{2}^{*}$ & $\beta_{0}$ & $\eta$ \\
\hline 0.2 & 500 & 0.4 & 100 & 494. & 99. & 0.209 & 1.204 \\
0.3 & 500 & 0.3 & 100 & 490. & 99. & 0.210 & 1.205 \\
0.4 & 500 & 0.2 & 100 & 494. & 99. & 0.209 & 1.204 \\
0.2 & 100 & 0.4 & 500 & 96. & 568. & 6.674 & 0.919 \\
0.3 & 100 & 0.3 & 500 & 96. & 558. & 6.462 & 0.927 \\
0.4 & 100 & 0.2 & 500 & 96. & 568. & 6.674 & 0.919
\end{tabular}

\subsection{Flat punch}

The flat punch solution is a particularly simple case to analyse, since the extent of contact is known, and inversion of Eq. (24) does not require iteration. Also, since $a$ is independent of indentation depth in this case, Eq. (27) shows that the indentation load depends linearly on displacement, and from Eq. (29) the apparent contact modulus is constant for a given punch. As a consequence, the analysis of load-displacement traces for any fixed punch radius does not reveal any depth-resolving information about the material properties of the system.

However, if the punch radius $a$ (we imply that it is normalised by layer thickness $h$, as all other linear dimensions) is allowed to vary, then the full range of the coated system response to be examined. This is illustrated in Fig. 8, where the apparent contact modulus is plotted against the relative contact radius, which in this context replaces the relative indentation depth. Simple qualitative analysis confirms that when $a / h \ll 1$, the zone of highest stresses is confined to a region in the vicinity of contact, and lies entirely inside the surface layer. Consequently, the apparent contact modulus can be expected to have the value close to that of the layer. In the other extreme, when a flat cylindrical punch has the radius which greatly exceeds the layer thickness, then the apparent contact modulus is likely to approach that of the substrate.

For very large punch radii we remark that in Fig. 8 some variation is observed in the apparent contact moduli. The source of this fluctuation is numerical instability linked to the large values of $a / h$. It can be eliminated, to a certain extent, by choosing a larger number of discretisation points. However, the calculation becomes too inefficient and prone to accumulation of errors. Proper asymptotic analysis would be the correct approach to this class of problems.

The detailed nature of the transition between the two extremes was determined using the solution method described in the previous section. The apparent contact modulus in this case is defined as

$E_{\text {flat }}^{*}(a)=\frac{P}{2 d a}$.

Two cases were considered: that of perfect bonding at the layer/ substrate interface, and that of frictionless adhesion, i.e. when only normal tractions are transmitted. The results show that the model response function based on the relative contact radius,

$E^{*}(a)=e_{2}^{*}+\frac{e_{1}^{*}-e_{2}^{*}}{1+\left(\frac{1}{\beta_{0}} \frac{a}{h}\right)^{\eta}}$

once again affords a good representation of the transition. The identified parameters $e_{1}^{*}, e_{2}^{*}$ lie close to the apparent contact modulus of the layer and substrate (see Table 4).

This last result suggests that the apparent contact modulus could be represented as a function of the relative contact radius for other indenter shapes as well. Indeed, the results for the conical indenter presented in Fig. 9 show a pattern similar to the previous plots (cf. also Tables 11 and 12).

For the sake of completeness, we list the results for apparent contact modulus and model response function parameter determination in the tables given in Appendix B.

\section{Conclusions}

We have presented the application of a simple model response function to the analysis of indentation of elastic coated systems. We consider the utility of this approach to lie in the simplicity with which the elastic response can be described over several decades of relative indentation depths or loads. The use of the model response function to fit the numerical results reveals the asymptotic behavior of the apparent contact modulus of coated systems.

It may be expected that in the extreme of very shallow indentation, $\beta \ll 1$, the corresponding parameter $e_{1}^{*}$ ought to approach the plane strain modulus of the coating $E_{1}^{*}$. Our results appear to confirm this expectation. Similarly, one might also expect that in the extreme of very deep indentation, $\beta \gg 1$, the corresponding parameter $e_{2}^{*}$ ought to approach the plane strain modulus of the substrate $E_{2}^{*}$. This statement is much more difficult to justify. It also appears that our results do not always follow this pattern. A definitive argument on this subject may only be mounted on the basis of detailed asymptotic analysis.

The elastic models used in the present study were chosen due to their elegance and compactness: they allow the analysis of parameter and indenter shape sensitivity in a way that is far more efficient than, for example, the use of finite element modeling. As a means of encapsulating the results of these modeling efforts in a form that is readily accessible and easy to visualize, we used once again the Model Response Function (MRF) approach. Previously this method has been applied to the consideration of experimental data on the depth-dependence of composite hardness.

The fundamental approach used in previous studies was to consider the expenditure of energy during indentation. This was chosen because energy analysis allows one to obviate the need for detailed complete analysis of deformation fields that in the case of specific material response can be quite laborious. Nevertheless,
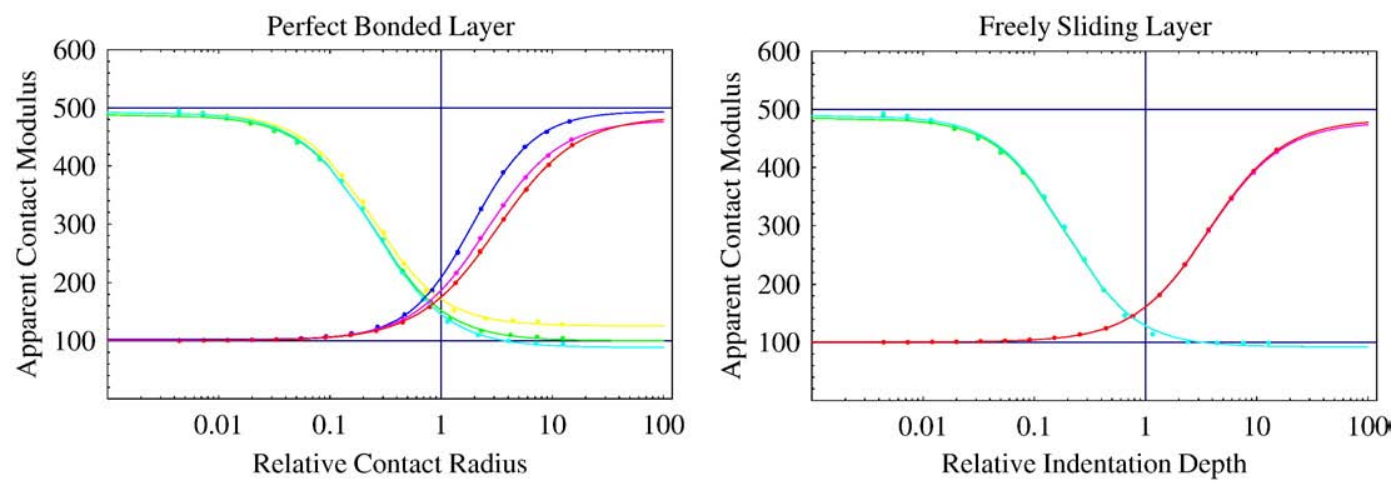

Fig. 9. The apparent contact modulus versus relative contact radius for perfect bonded and a freely sliding film respectively indented with a cone. 
elastic-plastic finite element modeling was also carried out, and very good agreement with energy-based predictions was obtained, enhancing the level off confidence in the choice of predictive model.

In the case of purely elastic material response, the present results demonstrate that a combined analytical-numerical treatment of the problem becomes possible, so that detailed characterization of the deformation and stress fields can be obtained. For that reason energy analysis is not employed here.

Another important conclusion that is substantiated and formulated in the present paper is that the same Model Response Function successfully applied to plasticity analysis (hardness-depth variation) can also be used to describe the apparent contact stiffness variation with depth. The physical meaning of the adopted function remains the same: it provides a compact, heuristic description of the relationship between important parameters of the indentation experiment.

\section{Acknowledgments}

We would like to express our gratitude to Dr Sean Yu of the Naval Research Lab, Washington, DC, for providing us with corrected formulas for the integral equation kernel, and for valuable discussions. AC wishes to thank the Royal Society of London for the provision of a visiting fellowship to enable him to visit Oxford, and Trinity College, Oxford, for their hospitality. AMK wishes to thank the Nuffield Foundation for the provision of the award NUF-NAL.

\section{Appendix A. Additional formulas for the elastic problem}

Let us introduce the following notations for different expressions involving the elastic moduli of the layer and of the substrate:

$$
\begin{aligned}
& \mu_{1}=\frac{E_{1}}{2\left(1+v_{1}\right)} \quad \mu_{2}=\frac{E_{2}}{2\left(1+v_{2}\right)} \quad \eta=\frac{a_{2} \mu_{1}}{a_{1} \mu_{2}} \quad \beta=\frac{\mu_{1}}{\mu_{2}} \\
& a_{1}=1-v_{1} \quad b_{1}=1-2 v_{1} \quad k_{1}=3-4 v_{1} \\
& a_{2}=1-v_{2} \quad b_{2}=1-2 v_{2} \quad k_{2}=3-4 v_{2} .
\end{aligned}
$$

The preceding expressions will be combined to form new symbols:

$$
\begin{aligned}
B_{1}= & 4 a_{1} a_{2}\left(r 1+b_{2} \beta\right) \beta \\
B_{2}= & -1-3 b_{2} \beta+\left(k_{2}-2 b_{2}^{2}\right) \beta^{2}+b_{2} k_{2} \beta^{3} \\
B_{3}= & 2 a_{2}\left(1+{ }_{2} k_{1}+2 b 2 k_{1} \beta+k_{2} \beta^{2}\right) \beta \\
B_{4}= & k_{1}+\left(-b_{2}+2 b 2 k_{1}+4 a_{1} a_{2}\right) \beta+\left(k_{1}+4 a_{1} a_{2} k_{2}+2 b_{1} b_{2}^{2}\right) \beta^{2} \\
& +b_{2} k_{2} \beta^{3} \\
B_{5}= & -1-\left(1+4 b_{2}\right) \beta+\left(1-2 b_{2}\right) k 2 \beta^{2}+k_{2}^{2} \beta^{3} \\
B_{6}= & k_{1}+\left(1+2 k_{1} k 2\right) \beta+\left(k 2+4 a 2 b 2 k 1+16 a_{1} a 2^{2}+2 b_{1} b_{2}^{2}\right) \beta^{2} \\
& +k_{2}^{2} \beta^{3} \\
C_{1}= & 4 a_{1}^{2}(1+b 2 \beta) \\
C_{2}= & 1+3 b_{2} \beta-\left(k_{2}-2 b_{2}^{2}\right) \beta^{2}-b_{2} k_{2} \beta^{3} \\
C_{3}= & k_{1}-\left(1-2 k_{1}\right) b_{2} \beta+\left(k 2+16 a_{1} a 2^{2}+2 b_{1} b_{2}^{2}\right) \beta^{2}+b_{2} k_{2} \beta 3 \\
C_{4}= & 4 a_{1}^{2}+4 a_{1}\left(2 a 2+a_{1} k_{2}\right) \beta+8 a_{1} a_{2} b_{1} \beta^{2} \\
C_{5}= & 1+\left(k_{2}+2 b_{2}\right) \beta-\left(1-2 b_{2}\right) k_{2} \beta^{2}-k_{2}^{2} \beta^{3} .
\end{aligned}
$$

Finally, for a perfectly bonded layer the function $g$ is expressed as:

$g_{b}=1-\frac{\left(B_{1}+B_{2} w+B_{3} \sinh ^{2} w\right) e^{-w}+\left(B_{4}+B_{5} w+B_{6} \sinh ^{2} w\right) \sinh w}{\left(C_{1}+C_{2} w^{2}+C_{3} \sinh ^{2} w\right) e^{-w}+\left(C_{4}+C_{5} w^{2}+B_{6} \sinh ^{2} w\right) \sinh w}$

and for a freely sliding layer as:

$g_{s}=1-\frac{\sinh ^{2} w+\eta(w+\sinh w \cosh w)}{w+\sinh w \cosh w+\eta\left(\sinh ^{2} w-w^{2}\right)}$.

\section{Appendix B. Additional tables}

Table 5

Computed apparent contact moduli for the indentation of a perfectly bonded layer with a sphere of radius $R=1$.

\begin{tabular}{lllllrll}
\hline$v_{1}$ & $E_{1}^{*}$ & $v_{2}$ & $E_{2}^{*}$ & $e_{1}^{*}$ & $e_{2}^{*}$ & $\beta_{0}$ & $\eta$ \\
\hline 0.2 & 500 & 0.4 & 100 & 484. & 109. & 0.115 & 0.593 \\
0.3 & 500 & 0.3 & 100 & 484. & 82. & 0.117 & 0.554 \\
0.4 & 500 & 0.2 & 100 & 491. & 69. & 0.121 & 0.535 \\
0.2 & 100 & 0.4 & 500 & 103. & 474. & 2.234 & 0.797 \\
0.3 & 100 & 0.3 & 500 & 102. & 426. & 3.024 & 0.734 \\
0.4 & 100 & 0.2 & 500 & 101. & 417. & 4.074 & 0.702 \\
\hline
\end{tabular}

\section{Table 6}

Computed apparent contact moduli for the indentation of a freely sliding layer with a sphere of radius $R=1$.

\begin{tabular}{lllllrll}
\hline$v_{1}$ & $E_{1}^{*}$ & $v_{2}$ & $E_{2}^{*}$ & $e_{1}^{*}$ & $e_{2}^{*}$ & $\beta_{0}$ & $\eta$ \\
\hline 0.2 & 500 & 0.4 & 100 & 482. & 71. & 0.076 & 0.565 \\
0.3 & 500 & 0.3 & 100 & 478. & 71. & 0.077 & 0.566 \\
0.4 & 500 & 0.2 & 100 & 482. & 71. & 0.076 & 0.565 \\
0.2 & 100 & 0.4 & 500 & 101. & 426. & 6.067 & 0.722 \\
0.3 & 100 & 0.3 & 500 & 101. & 424. & 6.042 & 0.722 \\
0.4 & 100 & 0.2 & 500 & 101. & 426. & 6.067 & 0.722 \\
\hline
\end{tabular}

Table 7

Computed apparent contact moduli for the indentation of a freely sliding layer with a sphere of radius $R=10$.

\begin{tabular}{lllllcll}
\hline$v_{1}$ & $E_{1}^{*}$ & $v_{2}$ & $E_{2}^{*}$ & $e_{1}^{*}$ & $e_{2}^{*}$ & $\beta_{0}$ & $\eta$ \\
\hline 0.2 & 500 & 0.4 & 100 & 495. & 62. & 0.738 & 0.526 \\
0.3 & 500 & 0.3 & 100 & 491. & 62. & 0.745 & 0.527 \\
0.4 & 500 & 0.2 & 100 & 495. & 62. & 0.738 & 0.526 \\
0.2 & 100 & 0.4 & 500 & 101. & 452. & 73. & 0.695 \\
0.3 & 100 & 0.3 & 500 & 101. & 449. & 73. & 0.695 \\
0.4 & 100 & 0.2 & 500 & 101. & 452. & 73. & 0.695 \\
\hline
\end{tabular}

Table 8

Computed apparent contact moduli for the indentation of a perfectly bonded layer with a sphere of radius $R=10$.

\begin{tabular}{lllllrrl}
\hline$v_{1}$ & $E_{1}^{*}$ & $v_{2}$ & $E_{2}^{*}$ & $e_{1}^{*}$ & $e_{2}^{*}$ & \multicolumn{1}{l}{$\beta_{0}$} & $\eta$ \\
\hline 0.2 & 500 & 0.4 & 100 & 494. & 99. & 1.147 & 0.549 \\
0.3 & 500 & 0.3 & 100 & 492. & 74. & 1.173 & 0.526 \\
0.4 & 500 & 0.2 & 100 & 497. & 63. & 1.217 & 0.515 \\
0.2 & 100 & 0.4 & 500 & 102. & 486. & 24.181 & 0.772 \\
0.3 & 100 & 0.3 & 500 & 101. & 434. & 32.131 & 0.717 \\
0.4 & 100 & 0.2 & 500 & 101. & 424. & 43.309 & 0.688 \\
\hline
\end{tabular}

\section{Table 9}

Computed apparent contact moduli for the indentation of a perfectly bonded layer with a sphere of radius $R=10$.

\begin{tabular}{llllllll}
\hline$v_{1}$ & $E_{1}^{*}$ & $v_{2}$ & $E_{2}^{*}$ & $e_{1}^{*}$ & $e_{2}^{*}$ & $\beta_{0}$ & $\eta$ \\
\hline 0.2 & 500 & 0.4 & 100 & 439. & 125. & 0.0153 & 0.733 \\
0.3 & 500 & 0.3 & 100 & 432. & 101. & 0.0162 & 0.700 \\
0.4 & 500 & 0.2 & 100 & 434. & 91. & 0.0172 & 0.685 \\
0.2 & 100 & 0.4 & 500 & 23. & 658. & 0.617 & 0.368 \\
0.3 & 100 & 0.3 & 500 & 70. & 561. & 0.854 & 0.450
\end{tabular}




\section{Table 10}

Computed apparent contact moduli for the indentation of a freely sliding layer with a sphere of radius $R=10$.

\begin{tabular}{llllllll}
\hline$v_{1}$ & $E_{1}^{*}$ & $v_{2}$ & $E_{2}^{*}$ & $e_{1}^{*}$ & $e_{2}^{*}$ & $\beta_{0}$ & $\eta$ \\
\hline 0.2 & 500 & 0.4 & 100 & 394. & 96. & 0.013 & 0.818 \\
0.3 & 500 & 0.3 & 100 & 391. & 96. & 0.014 & 0.820 \\
0.4 & 500 & 0.2 & 100 & 394. & 96. & 0.013 & 0.818 \\
0.2 & 100 & 0.4 & 500 & 90. & 514. & 1.269 & 0.550 \\
0.3 & 100 & 0.3 & 500 & 90. & 510. & 1.261 & 0.549 \\
0.4 & 100 & 0.2 & 500 & 90. & 514. & 1.269 & 0.550 \\
\hline
\end{tabular}

\section{Table 11}

Computed apparent contact moduli for the indentation of a freely sliding layer with a cone.

\begin{tabular}{llllllll}
\hline$v_{1}$ & $E_{1}^{*}$ & $v_{2}$ & $E_{2}^{*}$ & $e_{1}^{*}$ & $e_{2}^{*}$ & $\beta_{0}$ & $\eta$ \\
\hline 0.2 & 500 & 0.4 & 100 & 489. & 92. & 0.189 & 1.376 \\
0.3 & 500 & 0.3 & 100 & 485. & 92. & 0.190 & 1.377 \\
0.4 & 500 & 0.2 & 100 & 489. & 92. & 0.189 & 1.376 \\
0.2 & 100 & 0.4 & 500 & 100. & 483.11 & 3.690 & 1.283 \\
0.3 & 100 & 0.3 & 500 & 100. & 478.94 & 3.669 & 1.284 \\
0.4 & 100 & 0.2 & 500 & 100. & 483.11 & 3.690 & 1.283 \\
\hline
\end{tabular}

\section{Table 12}

Computed apparent contact moduli for the indentation of a perfectly bonded layer with a cone.

\begin{tabular}{lllllrll}
\hline$v_{1}$ & $E_{1}^{*}$ & $v_{2}$ & $E_{2}^{*}$ & $e_{1}^{*}$ & $e_{2}^{*}$ & $\beta_{0}$ & $\eta$ \\
\hline 0.2 & 500 & 0.4 & 100 & 491. & 125. & 0.242 & 1.367 \\
0.3 & 500 & 0.3 & 100 & 488. & 99. & 0.244 & 1.318 \\
0.4 & 500 & 0.2 & 100 & 492. & 88. & 0.248 & 1.296 \\
0.2 & 100 & 0.4 & 500 & 102. & 494. & 1.907 & 1.526 \\
0.3 & 100 & 0.3 & 500 & 100. & 479. & 2.568 & 1.300 \\
0.4 & 100 & 0.2 & 500 & 100. & 485. & 3.213 & 1.220 \\
\hline
\end{tabular}

\section{References}

[1] K.L. Johnson, Contact Mechanics, Cambridge University Press, 1985.

[2] G.M.L. Gladwell, Contact Problems in the Classical Theory of Elasticity, Sijthoff \& Noordhoff, 1980.

[3] J. Li, T.W. Chou, Elastic field of a thin-film/substrate system under an axisymmetric loading, Int. J. Solids Struct. 34 (34-35) (1997) 4463.

[4] N. Schwarzer, Arbitrary load distribution on a layered half space, ASME J. Tribol. $122(10)(2000) 672$.

[5] H.Y. Yu, S.C. Sanday, B.B. Rath, The effect of substrate on the elastic properties of films determined by the indentation test - axisymmetric Boussinesq problem, J. Mech. Phys. Solids 38 (6) (1990) 745.

[6] M. Lichinchi, C. Lenardi, J. Haupt, R. Vitali, Simulation of Berkovich nanoindentation experiments on thin films using finite element method, Thin Solid Films 312 (1998) 240.

[7] D. Tabor, The Hardness of Metals, Oxford University Press, 2000.

[8] M.F. Doerner, W.D. Nix, A method for interpreting the data from depth sensing indentation instruments, J. Mater. Res. 1 (4) (1986) 601.

[9] W.C. Oliver, G.M. Pharr, An improved technique for determining hardness and elastic modulus using load and displacement sensing instruments, J. Mater. Res. 7 (6) (1992) 1564

[10] N.N. Lebedev, I.S. Uflyand, PMM 22 (1958) 320.

[11] A.M. Korsunsky, M.R. McGurk, S.J. Bull, T.F. Page, On the hardness of coated systems, Surf. Coat. Technol. 99 (1998) 171.

[12] N. Tardieu, A. Constantinescu, On the determination of elastic coefficients from indentation experiments, Inverse Probl. 16 (3) (2000) 577.

[13] G.M. Pharr, A. Bolshakov, Understanding nanoindentation unloading curves, J. Mater. Res. 17 (2002) 2660.

[14] L. Solomon, Elasticité linèaire, Masson, Paris, 1968.

[15] A.M. Korsunsky, The influence of punch blunting on elastic indentation response J. Strain Anal. Eng. Des. 36 (2001) 391.

[16] S. Wolfram, The Mathematica Book, 4th ed.Cambridge University Press, 1999.

[17] D.A. Hills, D. Nowell, A. Sackfield, Mechanics of Elastic Contacts, ButterworthHeinemann, 1993.

[18] A. Constantinescu, A. Korsunsky, Elasticity with Mathematica, Cambridge University Press, 2007, 268 pp. 